\title{
Impact of Image-Space Resolution Modeling for Studies with the High-Resolution Research Tomograph
}

\author{
Florent C. Sureau ${ }^{1,2}$, Andrew J. Reader ${ }^{3}$, Claude Comtat ${ }^{1}$, Claire Leroy ${ }^{4}$, Maria-Joao Ribeiro ${ }^{1}$, Irène Buvat ${ }^{5}$, and \\ Régine Trébossen ${ }^{1}$ \\ ${ }^{1}$ Service Hospitalier Frédéric Joliot, I I BM, DSV, CEA, Orsay, France; ${ }^{2}$ Siemens Medical Solutions SAS, Saint-Denis, France; \\ ${ }^{3}$ School of Chemical Engineering and Analytical Science, University of Manchester, Manchester, United Kingdom; \\ ${ }^{4}$ INSERM-CEA UMR 797, Service Hospitalier Frédéric Joliot, Orsay, France; and ${ }^{5}$ INSERM-UPMC UMR 678, CHU \\ Pitié-Salpêtrière, Paris, France
}

Brain PET in small structures is challenged by low resolution inducing bias in the activity measurements. Improved spatial resolution may be obtained by using dedicated tomographs and more comprehensive modeling of the acquisition system during reconstruction. In this study, we assess the impact of resolution modeling $(\mathrm{RM})$ during reconstruction on image quality and on the estimates of biologic parameters in a clinical study performed on a high-resolution research tomograph. Methods: An accelerated list-mode ordinary Poisson ordered-subset expectation maximization (OP-OSEM) algorithm, including sinogram-based corrections and an experimental stationary model of resolution, has been designed. Experimental phantom studies are used to assess contrast and noise characteristics of the reconstructed images. The binding potential of a selective tracer of the dopamine transporter is also assessed in anatomic volumes of interest in a 5-patient study. Results: In the phantom experiment, a slower convergence and a higher contrast recovery are observed for RM-OP-OSEM than for OP-OSEM for the same level of statistical noise. RM-OP-OSEM yields contrast recovery levels that could not be reached without RM as well as better visual recovery of the smallest spheres and better delineation of the structures in the reconstructed images. Statistical noise has lower variance at the voxel level with RM than without at matched resolution. In a uniform activity region, RM induces higher positive and lower negative correlations with neighboring voxels, leading to lower spatial variance. Clinical images reconstructed with RM demonstrate better delineation of cortical and subcortical structures in both time-averaged and parametric images. The binding potential in the striatum is also increased, a result similar to the one observed in the phantom study. Conclusion: In high-resolution PET, RM during reconstruction improves quantitative accuracy by reducing the partial-volume effects.

\footnotetext{
Received Sep. 13, 2007; revision accepted Feb. 21, 2008.

For correspondence or reprints contact: Florent C. Sureau, Service Hospitalier Frédéric Joliot, 4 Place Général Leclerc, Orsay Cedex, France 91406.

E-mail: florent.sureau@cea.fr

COPYRIGHT ( 2008 by the Society of Nuclear Medicine, Inc.
}

Key Words: PET imaging; partial-volume effects; resolution modeling

J Nucl Med 2008; 49:1000-1008

DOI: 10.2967/jnumed.107.045351

PET is widely used in brain imaging to provide quantitative biologic images. However, it suffers from bias because of the partial-volume effect (PVE) caused by the low resolution of the images and from the noise because of the statistical nature of the processes involved.

Brain PET recently benefited from the development of scanners such as the ECAT high-resolution research tomograph (HRRT) (Siemens Molecular Imaging) (1). The HRRT is a dual-layer, crystal-detector scanner allowing a depth-of-interaction (DOI) measurement for the incident photons. It is characterized by an isotropic $2.5-\mathrm{mm}$ intrinsic spatial resolution. Spatial resolution in the reconstructed images varies radially and tangentially from about 2.5 to about $3 \mathrm{~mm}$ and axially from 2.5 to $3.5 \mathrm{~mm}$ in the $10-\mathrm{cm}$ field of view (FOV) covering most of the brain (2). Therefore, this tomograph dedicated to brain imaging, compared with whole-body tomographs with at best a 4.5-mm intrinsic resolution, reduces the biases caused by PVEs in the small cerebral regions involved in specific functional pathways.

However, the HRRT does not simultaneously offer sufficiently increased sensitivity to maintain a level of noise comparable to that of conventional tomographs. To limit statistical noise in dynamic studies, iterative algorithms with postreconstruction smoothing are usually required.

To avoid smoothing and thus further degrading resolution, new iterative reconstruction schemes with more comprehensive modeling of the acquisition system should be devised. This could be done by considering a less-approximate system matrix including, for instance, the depth-dependent resolution effect along a line of response (LOR). 
To avoid storing the large number of elements contained in the system matrix, several parameterizations of the system matrix have been proposed. Recent approaches tackled the problem by including only geometric effects in the system matrix and estimating resolution modeling (RM) either in the image space $(3,4)$ or in the projection space (5-9). The parameters of these models have been derived by analysis $(7)$ or by experiment $(3-5,9)$ or from Monte Carlo (MC) simulations $(6,10,11)$. In particular, these models have been used for small-animal scanners, where improved resolution is crucial (11).

RM in image space can be efficiently combined with listmode (LM) reconstruction in dynamic studies. Indeed, if resolution degradation is modeled in projection space, multiple lines of response should be forward-projected for a single bin, and a neighborhood of bins should be backprojected for each single event, leading to important losses in computation time. Standard acceleration techniques of the expectation maximization algorithm (by choosing angular subsets) are not efficient if RM is also angularly modeled.

This study, therefore, explores the impact on image quality and quantification of image-space RM for the HRRT. As resolution degradation in the FOV is limited for the HRRT (because of DOI capability), the proposed method is implemented as a shift-invariant convolution in image space. The approach is tested with physical phantoms, and its impact is assessed on parametric imaging in a clinical study.

\section{MATERIALS AND METHODS}

\section{Reconstruction for the HRRT}

The HRRT allows acquisition of data on 4.5 billion LORs irregularly sampled in projection space in LM format. These data are usually sorted into histograms and stored into regularly sampled sinograms, with each LOR being attributed to its closest sinogram element (bin). Angular axial compression is used to reduce the sampling level of the data and lower statistical uncertainty in the measured data. Estimates of scattered and random coincidences, as well as normalization and attenuation correction factors, are available as sinograms. A model of the acquisition process compatible with these binned correction factors has been developed and included in an LM algorithm.

The forward model for an $I$-dimensional (I-D)-acquired vector p (in which $I$ is the number of LORs) follows a Poisson distribution with mean $\langle\mathbf{p}>$ :

$$
<\mathbf{p}>=\mathbf{A N X H f}+\mathbf{s}+\mathbf{r},
$$

where $\mathbf{f}$ is a $J$-dimensional vector holding the coefficients of the spatial basis functions (used to represent the spatial radioactivity concentration), $\mathbf{H}$ is a $J \times J$ matrix modeling the point-spread function (PSF) in the image space (3), and $\mathbf{X}$ is an $I \times J$ matrix modeling forward projection along the LORs. $\mathbf{A}$ and $\mathbf{N}$ are $I \times I$ diagonal matrices holding, respectively, attenuation factors and the inverse of the LOR normalization factors, and $\mathbf{s}$ and $\mathbf{r}$ are the $I-D$ vectors of scattered and random coincidences that contribute to the expected data.
The binning process may be represented as an $R \times I$ matrix $\mathbf{B}$, where $R$ is the number of sinogram bins. This yields the following forward model:

$$
<\mathbf{p}_{\mathbf{B}}>=\mathbf{B A N X H f}+\mathbf{B s}+\mathbf{B r} .
$$

Because the HRRT normalization factors are measured using a direct normalization process in the sinogram space, they are available only as $R$-dimensional vectors, and further approximations need to be performed. Unlike other correction factors, the normalization factors are inherently high-frequency data (because of crystal-efficiency variations). The attribution to an LOR of the normalization factor corresponding to its closest sinogram bin is not valid and would generate artifacts due to incorrect matching of the resolution of the LOR forward-projected data and the resolution of the normalization matrix (12). The following approximation must be performed:

$$
\mathbf{B N X} \approx \mathbf{N}_{\mathbf{B}} \mathbf{X}_{\mathbf{B}}
$$

Eq. 3

where $\mathbf{X}_{\mathbf{B}}$ is an $R \times J$ matrix modeling forward projection along the center of the sinogram bins and $\mathbf{N}_{\mathbf{B}}$ is an $R \times R$ diagonal matrix holding normalization factors. This results in the following estimate:

$$
<\mathbf{p}_{\mathbf{B}}>=\text { BANXHf }+\mathbf{B s}+\mathbf{B r} \approx \mathbf{A}_{\mathbf{B}} \mathbf{N}_{\mathbf{B}} \mathbf{X}_{\mathbf{B}} \mathbf{H f}+\mathrm{Bs}+\mathbf{B r}
$$

where $\mathbf{A}_{\mathbf{B}}$ is an $R \times R$ diagonal matrix with the attenuation correction factors for each sinogram bin. This approximation implies that all LORs contributing to a bin are treated as a single LOR located at the center of the bin. Following the derivation of the OP-EM algorithm previously reported (13), the RM-OP-EM algorithm based on sinogram corrections may then be written as:

$$
\mathbf{f}^{k+1}=\mathbf{f}^{k} \frac{\mathbf{H}^{\mathbf{T}} \mathbf{X}_{\mathbf{B}}^{\mathbf{T}}\left\{\frac{\mathbf{B p}}{\mathbf{X}_{\mathbf{B}} \mathbf{H} \mathbf{f}^{k}+\left(\mathbf{A}_{\mathbf{B}} \mathbf{N}_{\mathbf{B}}\right)^{-1}(\mathbf{B s}+\mathbf{B r})}\right\}}{\mathbf{H}^{\mathbf{T}} \mathbf{X}_{\mathbf{B}}^{\mathbf{T}} \mathbf{N}_{\mathbf{B}} \mathbf{A}_{\mathbf{B}} \mathbf{1}_{R}}, \quad \text { Eq. } 5
$$

where $\mathbf{1}_{R}$ is an $R-D$ vector for which all elements are equal to 1 .

The matrix $\mathbf{B}$ may be seen as a linear operator aligning each LOR in the LM file to the center of the nearest sinogram bin.

The algorithm in Equation 5 may be implemented in list mode, preserving the temporal resolution of the LM data:

$$
\mathbf{f}^{k+1}=\mathbf{f}^{k} \frac{\mathbf{H}^{\mathbf{T}} \sum_{m=\mathbf{1}}^{L M} \mathbf{X}_{\mathbf{B}}^{\mathbf{T}}\left\{\frac{\mathbf{B} \boldsymbol{\delta}_{m}}{\mathbf{X}_{\mathbf{B}} \mathbf{H} \mathbf{f}^{k}+\left(\mathbf{A}_{\mathbf{B}} \mathbf{N}_{\mathbf{B}}\right)^{-1}(\mathbf{B s}+\mathbf{B r})}\right\}}{\mathbf{H}^{\mathbf{T}} \mathbf{X}_{\mathbf{B}} \mathbf{T}^{\mathbf{T}} \mathbf{N}_{\mathbf{B}} \mathbf{A}_{\mathbf{B}} \mathbf{1}_{R}}
$$

where $\boldsymbol{\delta}_{m}$ is an $I-D$ vector with a single 1 on the LOR corresponding to the event measured in the LM file, which contains LM events.

To accelerate reconstruction, only a subset of the LM data is used for each update of the spatial-basis coefficients. A fast and common approach in sinogram-based reconstructions is to use subsets with the azimuthal angle (14). This approach cannot be used in the case of an LM algorithm in which no histograms are calculated a priori. Another possibility is to consider temporal subsets of the LM data as previously described (15). The Poisson data would ensure complete independence between the subsets. 
Such ordered-subset (OS) algorithms are known not to converge but to generate image oscillations within an iteration due to discrepancies (related to noise, for instance) between subsets (14).

For sequential temporal subsets, variations between subsets might arise from the temporal evolution of the physiologic distribution of the tracer during an examination (or within a frame in a dynamic study). To limit discrepancies between data subsets, consecutive events in the LM file were assigned to different subsets; that is, the subsets were defined by gating on detected events.

When using temporal subsets, a scaling factor $S$ must be included in Equation 6:

$$
\mathbf{f}_{s+1}^{k}=\mathbf{f}_{s}^{k} \frac{\mathbf{H}^{\mathbf{T}} \sum_{m \in(L M)_{s}} S \mathbf{X}_{\mathbf{B}}^{\mathbf{T}}\left\{\frac{\mathbf{B}_{m}}{\mathbf{X}_{\mathbf{B}} \mathbf{H} \mathbf{f}_{s}^{k}+\left(\mathbf{A}_{\mathbf{B}} \mathbf{N}_{\mathbf{B}}\right)^{-1}(\mathbf{B s}+\mathbf{B r})}\right\}}{\mathbf{H}^{\mathbf{T}} \mathbf{X}_{\mathbf{B}}^{\mathbf{T}} \mathbf{N}_{\mathbf{B}} \mathbf{A}_{\mathbf{B}} \mathbf{1}_{R}},
$$

where the data are divided into $S$ temporal subsets, and $(L M)_{\mathbf{s}}$ corresponds to the $s$-th temporal subset and to $\mathbf{f}_{1}^{k+1}=\mathbf{f}_{s+1}^{k}$. This algorithm is referred to as RM-OP-OSEM in this article.

The factor $S$ simply scales up the reduced number of counts observed in a subset of the data, appropriately weighting the scattered and random events to the prompts.

The RM-OP-OSEM algorithm may be seen as an algorithm that simultaneously performs reconstruction and deconvolution (note that RM in the image space is closely related to the RichardsonLucy deconvolution algorithm (16)). As observed in deconvolution, reconstruction with RM is associated with a ringing artifact that appears near sharp intensity variations (17). Inappropriate kernels can generate the ringing artifacts (7). For RM-OP-OSEM with RM in image space, an MC simulation has shown that this artifact is accentuated by overmodeling the resolution effects (i.e., when the convolution kernel is too broad) and that the artifact still occurs when the convolution kernel perfectly matches the kernel used for simulating the resolution-degrading phenomena (3). On the other hand, when the kernel is underestimated, the resulting images displayed intermediate resolution but fewer ringing artifacts.

The ringing artifact is related to errors in the convolution kernel (due to its spatial truncation, for instance). In the case of one study (3), the model for the convolution kernel may not be expected to match exactly the PSF modeled in the MC simulation (as the projection/backprojection operators include interpolation steps, introducing further blurring). The ringing artifact is also related to imperfect matching between continuous convolution, followed by sampling and sampled convolution. The PSF of a tomograph acts like a low-pass filter, and a limited level of aliasing occurs in the sampled blurred image when the sampling interval is smaller than twice the full width at half maximum (FWHM) of the PSF. On the other hand, discrete deconvolution leads to more extensive aliasing in the deconvolved image.

\section{Implementation of the Algorithm}

All data were first binned using a span of 9 (span refers to the number of copolar angles that are summed on a Michelogram (18)). This operation is described by matrix $\mathbf{B}$ in Equations 2-7.

An experimental image-space model of resolution was derived from a point-source reconstruction. As described in one study (3), the rationale is to perform a reconstruction with an algorithm that inverts the geometric part ( $\mathbf{X}$ in our equations) to fit a model to $\mathbf{H}$ in the final image. A matched projector and backprojector were implemented using the Siddon algorithm (19) to speed up LM reconstruction.

A $1-\mathrm{mm}$ point source of about $37 \mathrm{MBq}$ of ${ }^{18} \mathrm{~F}$ was placed in air, $1 \mathrm{~cm}$ off the center of the transaxial plane. This point source was reconstructed using the algorithm without $\mathrm{RM}$ (without $\mathbf{H}$ in Eq. 7) but still including the random-events correction. Isotropic and stationary resolution kernels for the HRRT were then found through fitting the following functions:

$$
f(r)=\frac{1}{N}\{\exp (-\alpha r)+\beta\}
$$

$$
f(r)=\frac{1}{N}\left\{\beta \exp \left(-\alpha_{1} r\right)+(1-\beta) \exp \left(-\alpha_{2} r\right)\right\}
$$

where $N$ was calculated such that the kernel is normalized to 1 , and the fit parameters are $\alpha$ and $\beta$ for Equation 8 and $\alpha_{1}, \alpha_{2}$, and $\beta$ for Equation 9. The exponential with an offset model (Eq. 8) and the 2-weighted-exponential model (Eq. 9) were intended to model both the central fast-decaying part and the much slower decaying tails observed in the reconstructed images. The fitting/optimization process modeled the 3-dimensional convolution of the point source with the kernel (either Eq. 8 or Eq. 9), and the parameters of the kernel were estimated through least-squares fitting of the data with the model.

The resolution kernel was then implemented in the preceding LM OP-OSEM algorithm (the kernel provides the elements for matrix $\mathbf{H}$ ), using temporal subsets according to Equation 7.

To assess the impact of a stationary model of resolution on a tomograph with spatially variant resolution, 3 point sources of about a $0.4-\mathrm{mm}$ diameter each were placed at different axial locations in the FOV of the HRRT (offset by 1,5 , or $10 \mathrm{~cm}$ ). About 1.4 million events were acquired, and RM-OP-OSEM was used for reconstruction with RM described by either Equation 8 or Equation 9.

Because the truncation of the kernel leads to an imperfect model of resolution, its impact on the ringing artifact was also investigated. The quality of the deconvolution was assessed using the ratio between the activity in the periphery of the point source divided by all of the activity, defined according to the following formula:

$$
Q=\frac{[\boldsymbol{I}(\boldsymbol{i}, \boldsymbol{j})]_{30}-[\boldsymbol{I}(\boldsymbol{i}, \boldsymbol{j})]_{2}}{[\boldsymbol{I}(\boldsymbol{i}, \boldsymbol{j})]_{30}}
$$

where $[\boldsymbol{I}(\boldsymbol{i}, \boldsymbol{j})]_{2}$ and $[\boldsymbol{I}(\boldsymbol{i}, \boldsymbol{j})]_{30}$ correspond to the overall activity in a box of 2 -voxel width (i.e., the 8 central voxels, the best spatial resolution according to the Shannon theorem) and the overall activity in a box of 30-voxel width around the point, respectively. Ideally $\mathrm{Q}$ should be close to 0 , and a higher $\mathrm{Q}$ indicates the spreading of activity from the original position of the point source due to imperfect deconvolution.

Finally, a mini-imaging system (Deluxe Phantom; Data Spectrum) with hot inserts was filled with ${ }^{18} \mathrm{~F}$, and images were acquired on the HRRT at 2 different axial positions: off-centered by 0 and $4 \mathrm{~cm}$ (each acquisition of about 270 million events). This procedure investigated possible artifacts (ringing or deformation) in the image due to imperfect deconvolution. 


\section{Noise and Contrast Recovery Characterization}

The RM-OP-OSEM algorithm was compared with the manufacturer-supplied sinogram-based OP-OSEM algorithm, which does not include any specific RM for the HRRT (20). This algorithm does not use the Siddon algorithm but rather a pointlattice forward projector and backprojector with bilinear interpolation (21).

Datasets were acquired on the HRRT and reconstructed into $256 \times 256 \times 207$ images (isotropic voxel volume, $\sim 1.8 \mathrm{~mm}^{3}$ ) from LM data using RM-OP-OSEM. The datasets were also binned (using span 9) and reconstructed using the OP-OSEM algorithm. The scatter sinograms were estimated using the single-scatter simulation method (22), and random sinograms were derived from smoothed delayed-coincidence window data (23).

Reconstruction with these 2 algorithms was accelerated. The OP-OSEM algorithm was originally optimized for parallel reconstruction on a dedicated $\mathrm{GHz}$ processor (Xeon 3.0; Intel) (21). The RM-OP-OSEM algorithm was parallelized, and reconstruction was performed on a cluster (10 nodes of AMD OPTERON 2.4$\mathrm{GHz}$ quadri-processors).

A cylindric contrast phantom (inner length, $187 \mathrm{~mm}$; inner diameter, $196 \mathrm{~mm}$ ) filled with ${ }^{18} \mathrm{~F}$ (initial activity, $55 \mathrm{MBq}$ ) was scanned for $4 \mathrm{~h}$ on the HRRT. A cold transmission scan was performed the next day. This phantom included 4 hot spheres and 2 cold spheres of $0.5-20 \mathrm{~cm}^{3}$ embedded in a warm background (with an activity ratio of about 2.4 between the hot spheres and the background). The resulting LM file of about 3.5 billion events was then split into 36 pseudogated files (each second of data for every $36 \mathrm{~s}$ was assigned to a different subset), leading to 36 statistically independent realizations of the same object. Scatter and random sinograms were estimated for each of the 36 realizations.

Contrast recovery and noise characteristics were investigated using the following figures: a contrast recovery coefficient (CRC) for each sphere against background statistical noise (SN) for various numbers of iterations, mean and variance of the voxels over all realizations (the number of iterations was selected such that the 2 algorithms had similar levels of noise for the smallest hot sphere), and covariance of a central voxel after summing 10 planes in the background region above the spheres.

The summed images of the 36 realizations from RM-OPOSEM and OP-OSEM reconstructions were thresholded to obtain volumes of interest (VOIs) around the spheres. These VOIs were then eroded by 1 pixel to avoid PVEs due to sampling (boundary between structures inside a voxel). Background VOIs of the same volume as the spheres were defined by axially shifting the VOIs in the background region above the spheres so that these regions were located at the same radial distance from the center of the FOV. The CRC for hot and cold spheres was calculated for each realization $i$, using the following equations:

$$
\begin{gathered}
C R C_{\text {hot }, i}=\frac{S_{i} / B_{i}-1}{C R_{\text {ref }}-1} \\
C R C_{\text {cold }, i}=\frac{B_{i}-S_{i}}{B_{i}},
\end{gathered}
$$

where $C R C_{\text {hot }, i}$ and $C R C_{\text {cold }, i}$ correspond to the CRC of hot and cold spheres; $S_{i}$ and $B_{i}$ are the mean signal in the sphere and in the background, respectively; and $C R_{\text {ref }}$ is the independently measured (using a $\gamma$-well counter) contrast ratio between the hot spheres and the background. For each sphere, the overall $C R C$ and background $S N$ for each sphere were then found using:

$$
\begin{aligned}
C R C=\left\langle C R C_{i}>\right. & \text { Eq. } 13 \\
S N=\frac{\operatorname{std}\left(B_{i}\right)}{<B_{i}>}, & \text { Eq. } 14
\end{aligned}
$$

where $<>$ represents the mean and $s t d()$ the SDs across all realizations $i$.

The statistical significance of the CRC differences between the 2 algorithms was evaluated for similar levels of noise. A Kolmogorov-Smirnoff test with Lilliefors correction was first performed to assess normality of the samples, followed by an F test on equal variance between samples (both tests were rejected if values were below 5\%), and finally a 1-way ANOVA with repeated measures was conducted to obtain $P$ values.

\section{Clinical Protocol}

Five healthy volunteers $(32.6 \pm 4 \mathrm{y})$ were injected with ${ }^{11} \mathrm{C}$ PE2I $(292 \pm 48 \mathrm{MBq})$, a selective dopamine transporter (DAT) radioligand. A dynamic time series was acquired for $1 \mathrm{~h}(5 \times 60 \mathrm{~s}$, $5 \times 120 \mathrm{~s}, 2 \times 150 \mathrm{~s}$, and $8 \times 300 \mathrm{~s}$ successive frames) and reconstructed using the same protocol as described in the previous section. For each reconstruction, the number of iterations was selected so that the average activity in the central nuclei had converged in all frames.

The binding potential (BP) of ${ }^{11} \mathrm{C}-\mathrm{PE} 2 \mathrm{I}$ to the DAT was computed using a simplified reference tissue model with the cerebellum as the nonspecific binding region (24). The BP values were obtained in anatomic VOIs in which ${ }^{11} \mathrm{C}$-PE2I specifically binds to DAT (ventral striatum [VS], putamen [Pu], and caudate [Ca]). These VOIs were manually drawn on MR images registered to the PET images. The VOIs presented small volumes (between 4 and $7.5 \mathrm{~cm}^{3}$ ) and were expected to be particularly prone to PVEs that would influence their BP values.

\section{RESULTS}

\section{Experimental Data}

Experimental Resolution Modeling and Point Sources. The fit parameters for the kernels were found to be $\alpha=$ $10.7 \mathrm{~cm}^{-1}$ and $\beta=3.1110^{-5}$ for Equation 8 and $\beta=$ $0.023, \alpha_{1}=5.41 \mathrm{~cm}^{-1}$, and $\alpha_{2}=15.13 \mathrm{~cm}^{-1}$ for Equation 9. Figure 1 displays the fits obtained after convolution of the kernel with the size of the point source. The model described in Equation 9 better describes the tails but also the central part of the point source (the single-exponential fit has slightly elevated values in the central part to compensate for the difference in the tails, which it could not accommodate).

Figure 2 shows the impact of a stationary kernel on reconstruction of the miniphantom. Resolution was improved, and only limited deformation of the rods occurred. The images demonstrate the improvement in resolution after RM: the apparent width of the rods decreased, whereas the intensity in the central part increased. Overall, activity remained identical, because of a normalized resolution kernel. 
FIGURE 1. Fitted profiles after convolution with size of point source (zoom, $\times 2.5$; pixel size, $\sim 0.5 \mathrm{~mm})$. Kernels are displayed in linear (A) and logarithmic (B) scales.

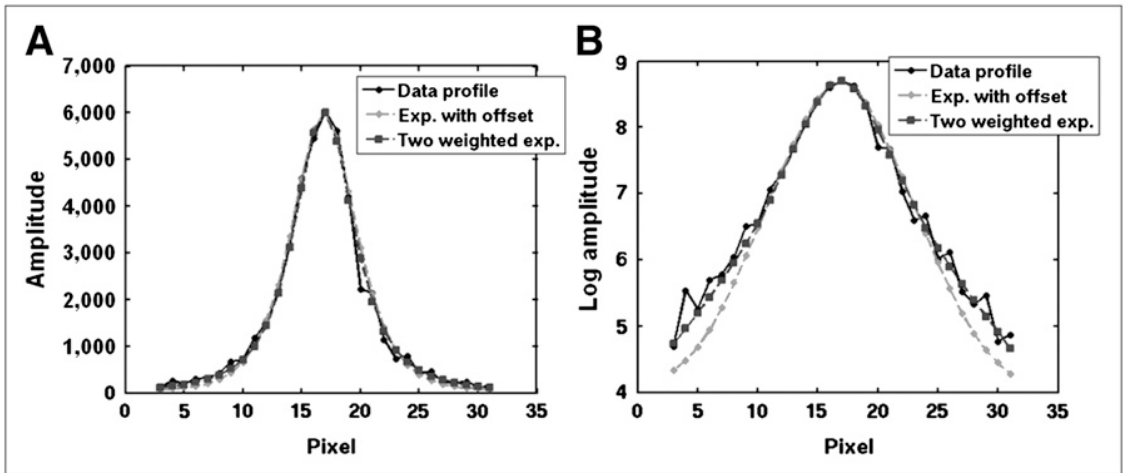

Truncated and imperfect resolution kernels affect reconstructed images. Ringing artifacts are found in images at locations corresponding to the periphery of the kernel and are reduced when the size of the kernel is increased (Fig. 3). The values of the periphery-to-total activity ratio defined in Equation 10 were calculated in the reconstructed images after convergence ( 15 iterations of 16 subsets) for various kernels and point-source positions and are reported in Table 1. The values show that Equation 9 performs better than Equation 8 and that the size of the kernel significantly affects the quality of deconvolution. Furthermore, only limited degradation occurred on the HRRT due to space-varying resolution compared with the size and model of the resolution kernel.

Contrast Phantom. Figure 4 compares the trade-off between SN and CRC for RM-OP-OSEM and OP-OSEM for the spheres in the contrast phantom. Slower conver-

\section{Without resolution modeling}
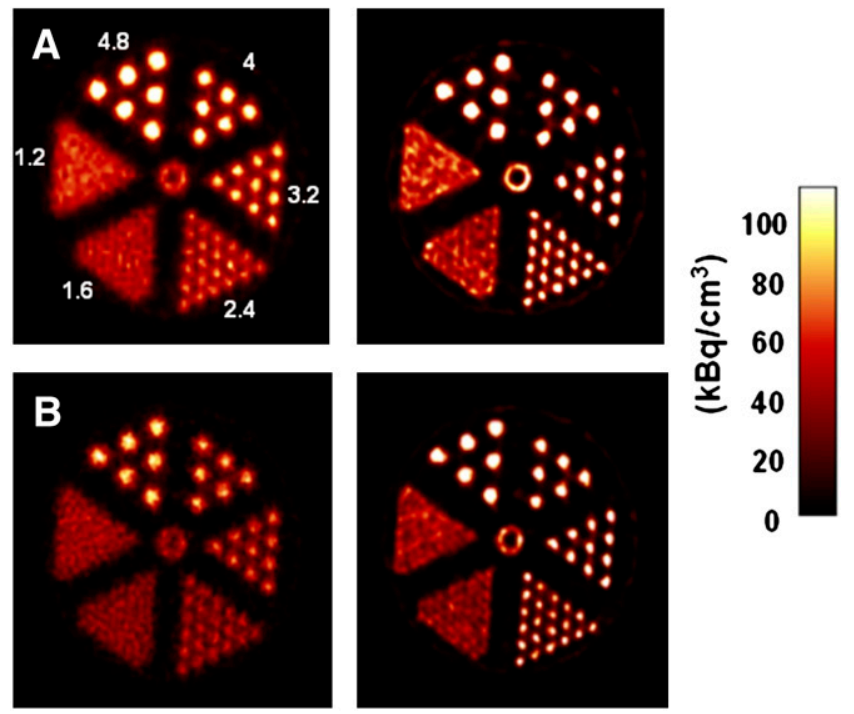

FIGURE 2. Qualitative impact of RM at 2 axial locations at center (A) and at $4 \mathrm{~cm}$ off center of FOV axially (B). Images without RM were reconstructed using 12 iterations of 16 subsets, whereas images with RM were reconstructed using 40 iterations of 16 subsets. gence occurs with RM-OP-OSEM (as seen for the 2-cm sphere, for instance; Fig. 4A). For a given level of noise, higher CRC is achieved with RM-OP-OSEM than with OP-OSEM, to levels that cannot be attained without RM. The difference in CRC between OP-OSEM images with 12 iterations and RM-OP-OSEM images with 12 or 16 iterations is significant for the largest 5 spheres $(P<0.05$ for the $1-\mathrm{cm}^{3}$ sphere, $P<0.001$ for the 4 largest spheres using ANOVA). Figure 4B shows that postfiltering simultaneously reduces image noise and CRC (the curves are shifted toward the bottom and the left) by degrading spatial resolution. This latter approach cannot match the improvements because of RM.

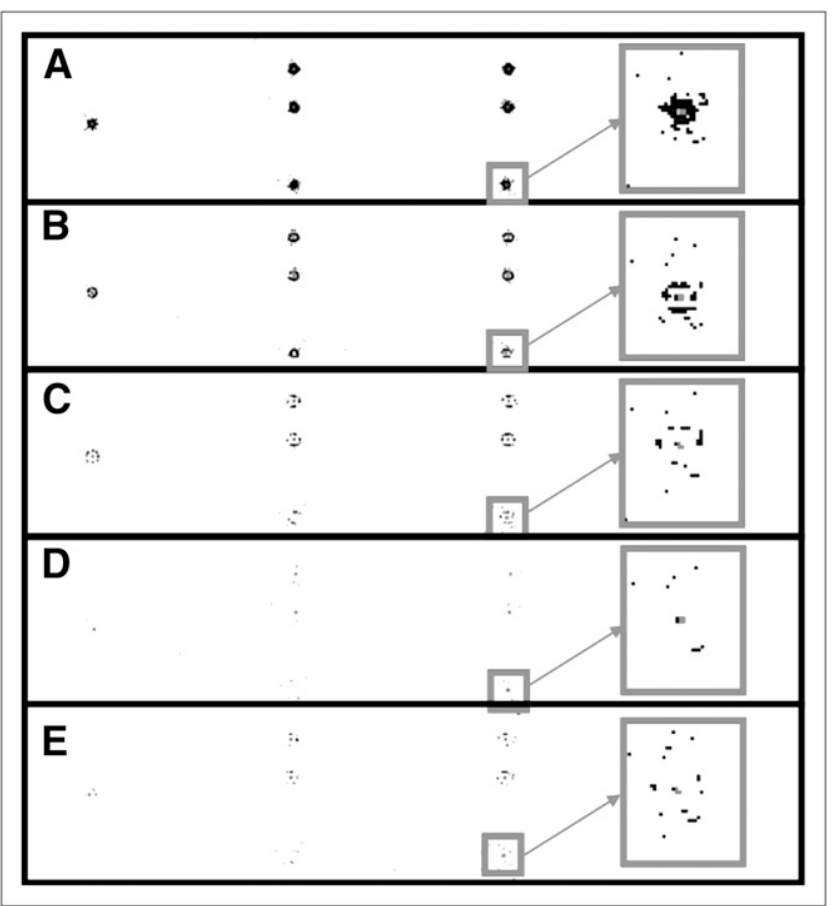

FIGURE 3. Axial, sagittal, and coronal planes of 3 point sources reconstructed with different resolution kernels (using 15 iterations of 16 subsets), with zoom on 1 point source in last column. Panels A, B, C, and D are reconstructed with 3-parameter kernel of size $3^{3}, 5^{3}, 9^{3}$, and $15^{3}$ voxels and $E$ with 2-parameter kernel of size $15^{3}$ voxels. Note that color scale was stretched to assist visualization of artifacts. 

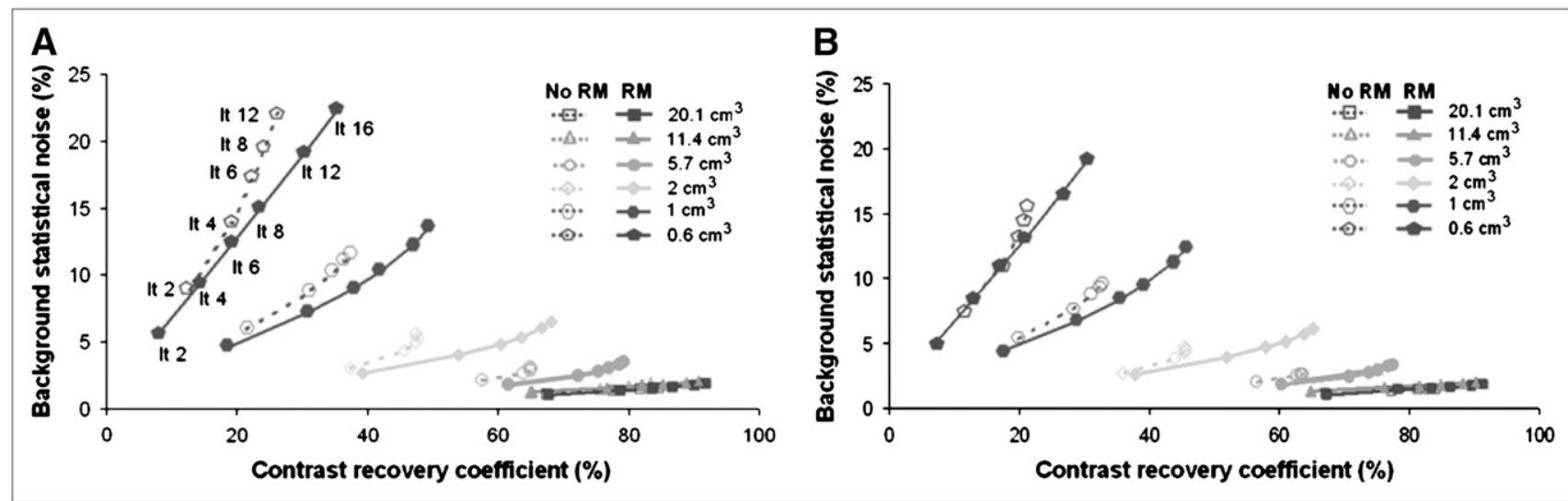

FIGURE 4. Impact of RM on CRC and noise properties, without (A) and with (B) postreconstruction smoothing. Each color corresponds to different sphere. RM-OP-OSEM curves are represented by filled symbols and solid lines and OP-OSEM curve by hollow symbols and dashed lines. Iteration numbers are shown for smallest sphere in $A$.

A visual inspection of the images in Figure 5A shows that RM-OP-OSEM offered better recovery of the smallest sphere than did OP-OSEM and better delineation of the contours between structures. This figure also shows that RM significantly reduced the voxel variance to a level comparable with the level that was obtained after postreconstruction smoothing with a 2-mm FWHM gaussian kernel, typically used for visualization. This is confirmed in Figure 5B, which also demonstrates that higher positive and lower negative correlations with adjacent voxels are observed when RM is used, leading to lower spatial variance.

Clinical Study. Time-averaged RM-OP-OSEM images obtained for 1 subject are displayed in Figure 6A and show improved delineation of the cortex and of the bindingspecific regions compared with OP-OSEM images. The average activities in these small regions were also higher than the activities in OP-OSEM images. The corresponding time-activity curves indicated an increase of activity concentration for late time frames in the small regions of interest (VS, Pu, and Ca increased by $13 \%, 15 \%$, and $12 \%$, respectively) compared with the activity concentrations derived from the OP-OSEM images as illustrated in Figure 6B. Unlike the small structures, the large nonspecific region (cerebellum) shows only a $2 \%$ increase in activity.

As reported in Table 2, BP values obtained with RM-OPOSEM were higher than those obtained with OP-OSEM. An increase was observed for all 5 subjects in all 3 VOIs, leading to a significant difference of $\mathrm{BP}$ values in these structures $(P<0.05$, Wilcoxon nonparametric test). This increase ranged from about $10 \%$ in the VS to about $20 \%-$ $25 \%$ in the $\mathrm{Ca}$ and $\mathrm{Pu}$.

$\mathrm{RM}$ also resulted in better delineation of cortical and subcortical structures for BP parametric maps. Postreconstruction filtering of the images before the parametric analysis leads to lower BP values due to degradation of spatial resolution.

\section{DISCUSSION}

This work demonstrates that RM in the reconstruction process improves spatial resolution (giving better delineation of structures), improves contrast recovery, and also improves the noise properties of the images.

A 20\%-40\% increase of contrast recovery was observed in a contrast phantom, and activity concentration estimates were increased by $12 \%-15 \%$ in the anatomic VOI in the clinical study. These results were confirmed in the parametric imaging study: BP values were significantly increased by $10 \%$ in the VS to $20 \%-25 \%$ in the $\mathrm{Ca}$ and $\mathrm{Pu}$. The smaller increase in the VS might be because of several phenomena: higher PVEs due to sampling in this smallest and thinnest region of the striatum that cannot be recovered by any of the 2 algorithms, significant spill-in from $\mathrm{Pu}$ and $\mathrm{Ca}$ in this region partly recovered with RM (as previously reported in a ${ }^{11} \mathrm{C}$-raclopride study using a geometric transfer matrix as correction for PVEs (25)), and possible errors in segmentation. These increased BP values are also consistent with a previous study on the HRRT (26) that reported an increase of about $30 \%$ in BP values for the

TABLE 1

Periphery-to-Total Activity Ratio (Eq. 10) of Point Source

\begin{tabular}{lccccc}
\hline $\begin{array}{c}\text { Kernel size } \\
\text { (offset axially) }\end{array}$ & \multicolumn{2}{c}{2 weighted exponentials } & & \multicolumn{2}{c}{ Single exponential } \\
\cline { 2 - 4 } & $3 \times 3 \times 3$ & $5 \times 5 \times 5$ & $9 \times 9 \times 9$ & $15 \times 15 \times 15$ & $15 \times 15 \times 15$ \\
\hline $\mathrm{cm}$ & 0.79 & 0.29 & 0.08 & 0.01 & 0.04 \\
$10 \mathrm{~cm}$ & 0.78 & 0.28 & 0.08 & 0.01 & 0.04 \\
\hline
\end{tabular}




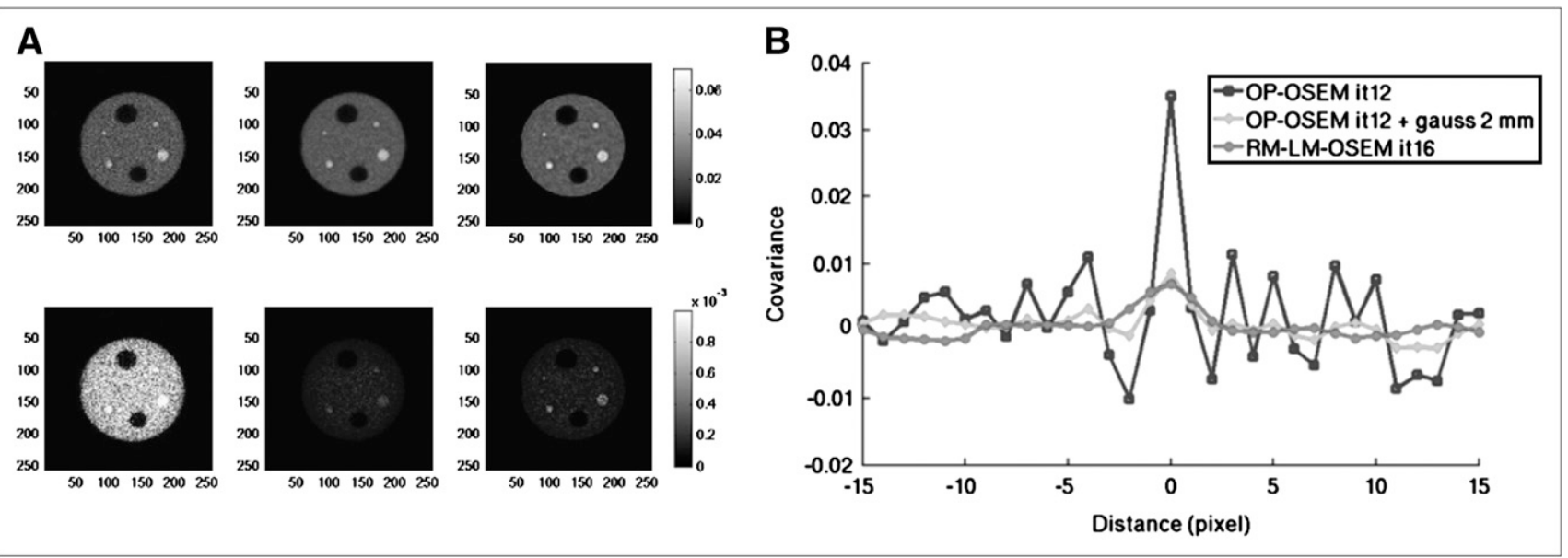

FIGURE 5. Impact of RM on noise properties. (A) Mean (first row) and variance images (second row) over all 36 realizations, for OP-OSEM (first column, 12 iterations of 16 subsets), OP-OSEM (12 iterations of 16 subsets) with 2-mm FWHM gaussian postreconstruction smoothing (second column), and RM-OP-OSEM (last column, 16 iterations of 16 subsets). (B) Covariance in summed planes on homogeneous background region.

$\mathrm{Ca}$ and $\mathrm{Pu}$, attributable only to the improved resolution of this tomograph (compared with BP values derived from the $\mathrm{HR}+$ tomograph).

For noise properties, RM increased positive correlations with adjacent voxels and decreased the negative correlations in a homogeneous region as previously reported (27). These results further indicate that RM has a significant impact on image quality by increasing spatial homogeneity in uniform activity concentration regions while increasing contrast recovery in small structures. In this work, we did not study the impact of data noise on RM. For the clinical study, we followed the protocol defined previously (26) (with frame duration of at least $1 \mathrm{~min}$; that is, about 8 million events at least per frame). The data did not require any postreconstruction smoothing, and thus resolution was not degraded after reconstruction. The advantages of RM are still impaired by data noise, and other reconstruction techniques need to be devised to work at lower count studies.

The proposed method used a simple experimentally derived model of resolution. First, the spatial resolution was chosen to be spatially invariant because the HRRT offers DOI measurement and consists of detector panels that limit spatial-resolution degradation over the FOV. This first hypothesis is reasonable for this tomograph. For a scanner with a more significant degradation of resolution,

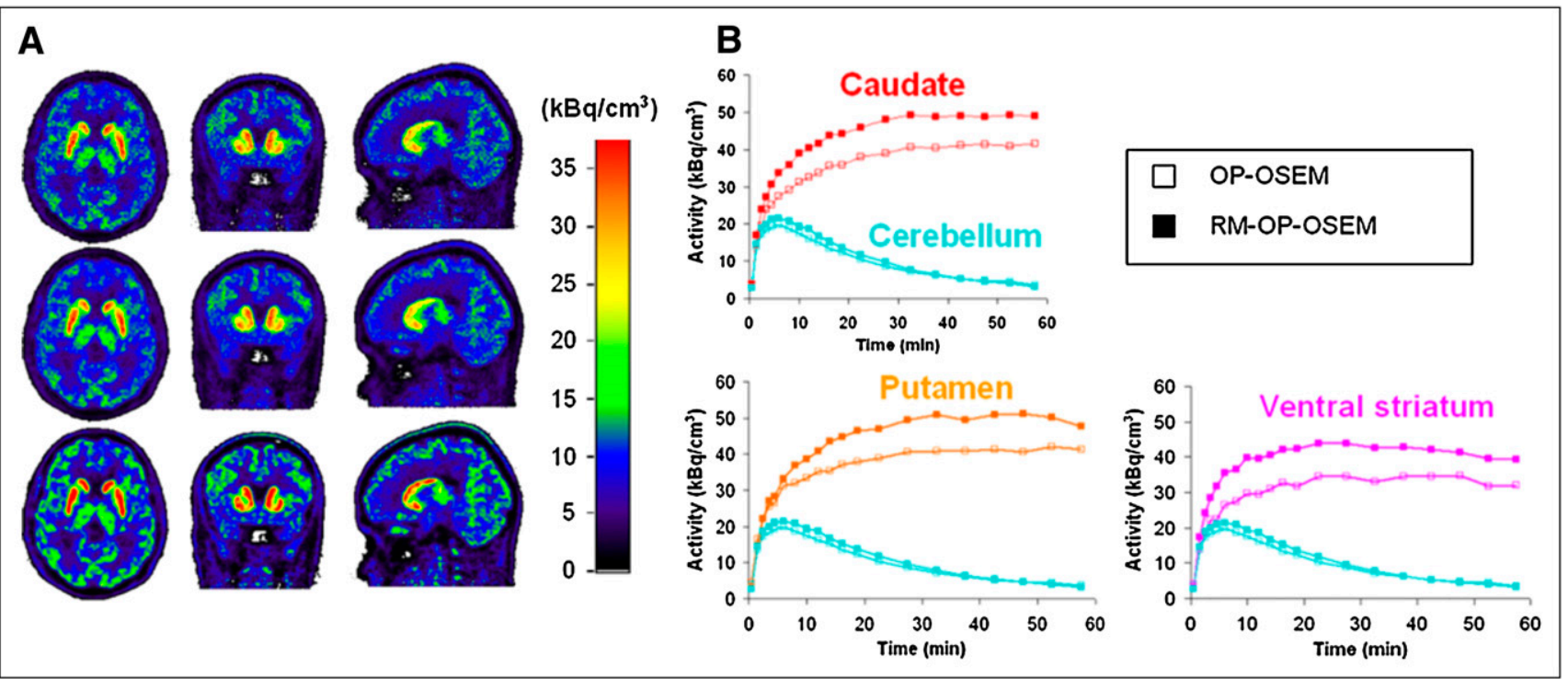

FIGURE 6. Time-averaged images (A) and corresponding VOI time-activity curves (B) for subject. In A, images across striatum were reconstructed with OP-OSEM (first row, 12 iterations of 16 subsets), OP-OSEM (12 iterations of 16 subsets) followed by a postreconstruction smoothing with 2-mm FWHM gaussian kernel (second row), and RM-OP-OSEM (last row, 15 iterations of 16 subsets). 
TABLE 2

BP Values in Anatomic Structures for All 5 Subjects

\begin{tabular}{lrcc}
\hline \multicolumn{1}{c}{ Parameter } & Caudate & Putamen & Ventral striatum \\
\hline Without RM & $9.11(0.6)$ & $10.25(1.3)$ & $7.1(0.8)$ \\
With RM & $11.34(0.7)$ & $12.60(1.7)$ & $7.8(1.1)$
\end{tabular}

Corresponding SDs are in parentheses.

more complex models taking into account spatially variant anisotropic resolution may be used in RM techniques operating in image space, as previously demonstrated (4).

However, the major advantage of using a shift-invariant resolution kernel is the ability to perform the convolution step in the Fourier domain to speed up the reconstruction process, which is crucial when the resolution kernel is not separable. The reconstruction of 1 frame in the clinical examination lasted about $1.5 \mathrm{~h}$ for 12 iterations of 16 subsets of the OP-OSEM algorithm and $4 \mathrm{~h}$ for 16 iterations of 16 subsets of the RM-OP-OSEM algorithm. Early tests show that reconstruction of typical scans would be accelerated by a factor of $2-3$, using fast Fourier transforms compared with image-based 3-dimensional convolution.

In this work, we focused only on image improvements enabled by RM. LM reconstruction with Siddon projectors was chosen to enable fast reconstructions in low-count dynamic examinations. In particular, RM-OP-OSEM does not take advantage of the higher spatial resolution of LM data because it uses binned sinograms for the corrections. However, we found in a simulation that span-3 or span-9 data binning resulted in only limited spatial-resolution degradation for the HRRT and that RM had a greater impact on resolution (12).

Although alternative component-based techniques have been proposed (28) to derive LOR normalization estimates from low-scan-time acquisitions, corrections using binned sinograms are still widely used. Implementing these techniques would be beneficial for the RM-OP-OSEM algorithm, because one would obtain slightly better spatial resolution and realigning of LORs would no longer be necessary.

Imperfect deconvolution was studied using a simple criterion showing that the choice of the number of voxels used to represent the kernel is crucial to limit ringing artifacts during reconstruction. Throughout iterations, deconvolution errors cannot be distinguished from reconstruction errors as RM is within the system matrix. When images converge, these errors can be further estimated by computing the error spectrum related to the ratio between the true (inverse filter when it exists) and the modeled deconvolution filters (29) or by using the approach described by Qi et al. (30) (it would require inversion of the Fisher information matrix, typically containing $10^{7} \times 10^{7}$ elements for the HRRT).
In addition, we did not study the effects of nonlinearities because of the use of the EM algorithm. In particular, resolution in the air (infinite contrast) is known to be better than resolution in a warm background (2). However, it is safer to underestimate the resolution rather than to overestimate it, as shown by Reader et al. (3): the former leads to a reconstructed image with blur (but less blur than without resolution modeling), whereas the latter leads to artifacts.

In this work, early stopping of the EM algorithm was used to "regularize" the reconstructions, and the impact of RM for given levels of noise or contrast was considered. In the clinical study, the stopping criterion was chosen so that the signal in the anatomic VOIs had converged for each frame. Even if other stopping criteria are considered (e.g., as described by Selivanov et al. (7)), it seems that RM should be included in maximum a posteriori algorithms. This approach has already been used and has proved beneficial (31). Early stopping of the reconstruction algorithm implies that the signal has not converged at the voxel level. The parametric maps derived from the reconstructed images can thus be used to investigate potential inhomogeneities in subregions but not to provide a parametric analysis at the voxel level.

\section{CONCLUSION}

An iterative algorithm including experimental shiftinvariant RM has been developed to take into account the resolution characteristics of the HRRT, and the resulting improvements in contrast recovery of small structures and noise properties in images have been demonstrated. RM also significantly affected the quantification of compartmental parameters used in kinetic modeling. This approach makes greater use of the high spatial resolution available with new tomographs, which is required for clinical dynamic brain PET.

\section{ACKNOWLEDGMENTS}

We are grateful to Christian Michel and Siemens Molecular Imaging for providing information and advice about binning and reconstruction on the HRRT. We thank Vincent Brulon and Yoann Fontyn for their precious help in designing and performing the experiments.

\section{REFERENCES}

1. Sossi V, de Jong H, Barker W, et al. The second generation HRRT: a multi-centre scanner performance investigation. IEEE Nucl Sci Symp Conf Record. 2005;4: 2195-2199.

2. de Jong HWAM, van Velden FHP, Kloet RW, Buijs FL, Boellaard R, Lammertsma AA. Performance evaluation of the ECAT HRRT: an LSO-LYSO double layer high resolution, high sensitivity scanner. Phys Med Biol. 2007;52: 1505-1526.

3. Reader AJ, Julyan P, Williams H, Hastings D, Zweit J. EM algorithm system modeling by image-space techniques for PET reconstruction. IEEE Trans Nucl Sci. 2003;50:1392-1397.

4. Rahmim A, Lenox M, Michel C, Reader A, Sossi V. Space-variant and anisotropic resolution modeling in list-mode EM reconstruction. IEEE Nucl Sci Symp Conf Record. 2003;5:3074-3077. 
5. Panin VY, Kehren F, Michel C, Casey M. Fully 3-D PET reconstruction with system matrix derived from point source measurements. IEEE Trans Med Imaging. 2006;25:907-921.

6. Alessio A, Kinahan P, Lewellen T. Modeling and incorporation of system response functions in 3-D whole body PET. IEEE Trans Med Imaging. 2006;25: 828-837.

7. Selivanov V, Picard Y, Cadorette J, Rodrigue S, Lecomte R. Detector response models for statistical iterative image reconstruction in high resolution PET. IEEE Trans Nucl Sci. 2000;47:1168-1175.

8. Mumcuoglu EU, Leahy RM, Cherry SR, Hoffman E. Accurate geometric and physical response modelling for statistical image reconstruction in high resolution PET. IEEE Nucl Sci Symp. 1996:1569-1573

9. Johnson C, Yan Y, Carson R, Martino R, Daube-Witherspoon M. A system for the 3D reconstruction of retracted-septa PET data using the EM algorithm. IEEE Trans Nucl Sci. 1995;42:1223-1227.

10. Qi J, Leahy RM, Hsu C, Farquhar T, Cherry S. Fully 3D Bayesian image reconstruction for the ECAT EXACT HR+. IEEE Trans Nucl Sci. 1998;45: 1096-1103.

11. Qi J, Leahy RM, Cherry SR, Chatziioannou A, Farquhar TH. High resolution 3D Bayesian image reconstruction using the microPET small-animal scanner. Phys Med Biol. 1998;43:1001-1013.

12. Reader AJ, Sureau FC, Comtat C, Buvat I, Trebossen R. List-mode reconstruction with system modeling derived from projections. IEEE Nucl Sci Symp Conf Record. 2005;3:1832-1836.

13. Politte DG, Snyder DL. Corrections for accidental coincidences and attenuation in maximum-likelihood image reconstruction for positron-emission tomography. IEEE Trans Med Imaging. 1991;10:82-89.

14. Hudson H, Larkin R. Accelerated image reconstruction using ordered subsets of projection data. IEEE Trans Med Imaging. 1994;13:601-609.

15. Reader AJ, Erlandsson K, Flower MA, Ott RJ. Fast accurate iterative reconstruction for low-statistics positron volume imaging. Phys Med Biol. 1998;43: $835-846$.

16. Lucy LB. An iterative technique for the rectification of observed distributions. Astron J. 1974;79:745-754.

17. Snyder D, Miller M, Thomas LJ, Politte D. Noise and edge artifacts in maximum-likelihood reconstructions for emission tomography. IEEE Trans Med Imaging. 1987;6:228-238.
18. Defrise M, Kinahan P. Data acquisition and image reconstruction for 3D PET. In: Bendriem B, Townsend DW, eds. The Theory and Practice of 3D PET. Norwell, MA: Kluwer; 1998.

19. Siddon RL. Fast calculation of the exact radiological path for a threedimensional CT array. Med Phys. 1985;12:252-255.

20. Comtat C, Bataille F, Michel C, et al. OSEM-3D reconstruction strategies for the ECAT HRRT. IEEE Nucl Sci Symp Conf Record. 2004;6:3492-3496.

21. Jones J, Jones W, Kehren F, et al. SPMD cluster-based parallel 3D OSEM. IEEE Trans Nucl Sci. 2003;50:1498-1502.

22. Watson C, Newport D, Casey M. A single scatter simulation technique for scatter correction in 3D PET. In: Grangeat P, Amans J-L, eds. Three-Dimensional Image Reconstruction in Radiology and Nuclear Medicine. Dordrecht: Kluwer Academic Publishers; 1996.

23. Byars L, Sibomana M, Burbar Z, et al. Variance reduction on randoms from coincidence histograms for the HRRT. IEEE Nucl Sci Symp Conf Record. 2005;5: 2622-2626.

24. Lammertsma AA, Hume SP. Simplified reference tissue model for PET receptor studies. Neuroimage. 1996;4:153-158.

25. Mawlawi O, Martinez D, Slifstein M, et al. Imaging human mesolimbic dopamine transmission with positron emission tomography: I. Accuracy and precision of $\mathrm{D}_{2}$ receptor parameter measurements in ventral striatum. J Cereb Blood Flow Metab. 2001;21:1034-1057.

26. Leroy C, Comtat C, Trebossen R, Syrota A, Martinot JL, Ribeiro MJ. Assessment of ${ }^{11} \mathrm{C}$-PE2I binding to the neuronal dopamine transporter in humans with the high-spatial-resolution PET scanner HRRT. J Nucl Med. 2007;48:538-546.

27. Rahmim A, Cheng J, Sossi V. Improved noise propagation in statistical image reconstruction with resolution modeling. IEEE Nucl Sci Symp Conf Record. 2005;5:2576-2578.

28. Bai B, Li Q, Holdsworth $\mathrm{CH}$, et al. Model-based normalization for iterative 3D PET image reconstruction. Phys Med Biol. 2002;47:2773-2784.

29. Lagendijk R, Biemond J, Boekee D. Regularized iterative image restoration with ringing reduction. IEEE Trans Acoust Speech Signal Process. 1988;36:1874-1888.

30. Qi J, Huesman RH. Effect of errors in the system matrix on maximum a posteriori image reconstruction. Phys Med Biol. 2005;50:3297-3312.

31. Bataille F, Comtat C, Jan S, Sureau FC, Trebossen R. Brain PET partial-volume compensation using blurred anatomical labels. IEEE Trans Nucl Sci. 2007;54: 1606-1615. 